\title{
La excepción de eficiencia
} económica. Análisis de su viabilidad en el régimen de competencia colombiano, desde la óptica del derecho económico ${ }^{1}$

\author{
MARLON IVÁN MALdONAdO NARVÁEZ
}

\section{RESUMEN}

El presente artículo analiza la excepción de eficiencia económica prevista en la Ley 1340 de 2009, a la luz de los aspectos económicos previstos en la Constitución Política de Colombia, en especial relación con las previsiones del artículo 333, y el mantenimiento de las políticas propias del Estado Social de Derecho. En especial, en la forma como la posibilidad de libertad de empresa prevista en virtud del mandato constitucional choca con los fines sociales y del ordenamiento jurídico del cual el Estado es garante, en procura del mantenimiento del equilibrio económico, del correcto desarrollo de la competencia empresarial, y por último, pero no menos importante, de la protección de los consumidores.

Palabras Clave. Excepción de Eficiencia Económica, Libre Competencia, Integración Empresarial, Posición de Dominio, Constitución Económica.

1 Para citar el artículo: Maldonado, M. (2015). La excepción de eficiencia económica. Análisis de su viabilidad en el regimen de competencia colombiano, desde la óptica del derecho económico, en Revista Con-texto, n. ${ }^{\circ} 44$, pp. 117-129. DOI: https://doi.org/10.18601/01236458.n44.06

2 Profesor de Derecho Económico, Derecho Comercial y Sociedades y Contratación Internacional, de la Universidad de Boyacá. Magíster en Derecho Empresarial de la Universidad Autónoma de Barcelona. Correo e: mmaldonadon@hotmail.com 


\section{ECONOMIC EFFICIENCY. ANALYSIS OF THEIR VIABILITY IN THE COLOMBIAN COMPETITION REGIME, FROM THE PERSPECTIVE OF ECONOMIC LAW}

\section{ABSTRACT}

This article analyzes the economic efficiency, under Law 1340 of 2009, in light of the economic aspects provided in the Constitution of Colombia, especially regarding the provisions of Article 333, and the maintaining of policies Rule of Law. Especially the way how possibility of free enterprise provided under constitutional mandate, collides with social goals of the State and the legal system which the same state is guarantor, in pursuit of maintaining economic balance, the proper conduct of business competition and last but not least the protection of consumers.

Keywords: Economic Efficiency, Competition, Business Integration, Dominance, Economic Constitution.

\section{INTRODUCCIÓN}

A lo largo del último año, hemos visto cómo la Superintendencia de Industria y Comercio ha venido estableciendo sanciones a los diferentes grupos empresariales y sectores económicos por la realización de conductas que afectan directamente no solo la libre competencia, sino también al consumidor, con lo cual es posible afirmar que afectan a la economía en general.

Esta situación no fuera de mayor importancia, si dentro de la Constitución Política de 1991 no se hubiese desarrollado un marco, de acuerdo con el cual es tarea del Estado velar, de una parte, por el mantenimiento de las diferentes garantías sociales, entre ellas el derecho a la igualdad. Paralelo a lo anterior, corresponde al Estado, como director de la economía nacional, establecer las leyes que faciliten el crecimiento y desarrollo económico, con fundamento en la legalidad, igualdad y justicia, como medio generador de seguridad jurídica para favorecer el crecimiento del tejido empresarial y la competitividad.

Con la expedición en Colombia de la Ley 155 de 1959, se empezó a hablar de un tema nuevo para el país, como lo era la prohibición de las prácticas comerciales restrictivas. Norma jurídica que podía entenderse bajo el contexto histórico de la época, como una respuesta al movimiento mundial en pro de la reactivación de la economía de la postguerra a través del desarrollo de la industria, el cual promovía la claridad del mercado a través de evitar cualquier práctica orientada a la formación de monopolios.

Sin embargo, más allá de la verificación de todos los aspectos positivos de la misma, y de que supuso un gran avance para un Estado como el colombiano, en el cual el tema no se encontraba ni siquiera previsto dentro de la Constitución vigente para la fecha de la promulgación de la norma ${ }^{3}$, su aplicabilidad práctica era prácticamente nula, ya que 
no existía como tal un gran tejido empresarial en el cual se requiriera de una vigilancia especial para evitar la realización de prácticas que pudieran poner en peligro a los distintos competidores del mercado.

Si bien en Colombia se siguió legislando al respecto ${ }^{4}$, no fue hasta después de 1991, con la expedición de una nueva Constitución Política, que supuso la modificación de la estructura del Estado, y al mismo tiempo obligó a examinar todas las normativas anteriores a la fecha de su promulgación, a efectos de verificar si estas se acogían o no al espíritu de la nueva Carta Política.

No obstante, muchas de las normas que se han desarrollado han hecho más énfasis en los aspectos económicos, situación de gran importancia para el crecimiento nacional, y no han tenido del todo en cuenta los aspectos jurídicos asociados a estas, con lo cual se favorece un clima de incertidumbre y desigualdad, que contraría el espíritu de la Carta Política.

Ya sea por desconocimiento, o por la importación de normas extranjeras al país, el hecho es que su aplicación no siempre puede ofrecer los resultados que se han obtenido en otras latitudes, o no garantizar el pleno de las garantías constitucionales, que se desarrollan en medio de un Estado de corte intervencionista.

Un ejemplo de tales normas lo podemos encontrar en la denominada excepción de eficiencia, la cual si bien no ha tenido mayor aplicación en el país, de ponerse de moda su uso, podría afectar la economía y, al mismo tiempo, generaría un clima de mayor incertidumbre, toda vez que no establece con claridad un criterio unificado para su otorgamiento, sino que en ciertos aspectos responde más a valoraciones subjetivas que a un proceso taxativamente señalado en igualdad de condiciones para todos los casos.

\section{LA CONSTITUCIÓN ECONÓMICA Y LA PROTECCIÓN DE LA COMPETENCIA}

La denominación de Estado Social de Derecho introducida por la Constitución Política de 1991 ha obligado al Estado a armonizar, de una parte, las políticas económicas y las sociales, de tal manera que las primeras sirvan para generar los recursos requeridos para garantizar las garantías propias de los coasociados en materia social. Al mismo tiempo supone, y de manera especial entre otros, la defensa del derecho a la igualdad de las personas en el territorio nacional ${ }^{5}$.

para solucionar cualquier situación que pudiese afectar el equilibrio económico, mas no hacía una referencia directa a los monopolios o a cómo una concentración empresarial podría ser nociva para la libre competencia.

4 En este sentido, puede verse a Alfonso Miranda Londoño, quien aporta una descripción de las diferentes normas que se promulgaron en el país sobre el tema, hasta la expedición del Decreto 2153 de 1992. Miranda londoño, Alfonso. "Origen y evolución del derecho de la competencia en Colombia, la Ley 155 de 1959 y su legado", en Revista del Derecho de la Competencia. Enero-diciembre, 2011, vol. 6, n. ${ }^{\circ}$ 6, pp. $65-148$.

5 Cortés Zambrano, Sonia Patricia. "El estado, la constitución y la economía de mercado", en Revista virtual vía inviniendi et iudicandi. Universidad Santo Tomás. Disponible en Internet: http://numanterioresviei.usta.edu.co/articulos/edi9/04-Sonia-Patricia-Cortez-Zambrano/EL\%20ESTADO,\%20LA\%20CONSTI- 
El paso de un modelo de una participación altamente centralista, con un gasto social realmente pequeño y con una protección arancelaria notoria, a un modelo aperturista, con el compromiso de aumentar el gasto público social, el aumento de la descentralización y una mayor libertad económica ${ }^{6}$, han supuesto para el Estado la adopción de un sinnúmero de medidas para adaptarlo a dichos compromisos.

Se trataba de preparar al país para una economía más abierta, una economía globalizada en la cual se facilitara el flujo de capitales nacionales y extranjeros en igualdad de condiciones $^{7}$, y como medio ideal para generar un crecimiento económico sostenido, que permitiera al Estado avanzar hacia la consecución de sus objetivos.

Para la realización de tales objetivos, la Constitución Política de 1991 consagró valores a tener en cuenta, pero no estableció un modelo económico puntual para seguir. Como tal, dejó abierta la posibilidad de usar modelos diversos que se ajustaran a las realidades político-económicas del país, aspecto que en realidad tiene plena lógica ante la dificultad que supone sufragar, para una economía como la colombiana, todos los gastos requeridos para el cumplimiento de la teleología de la Carta en lo que tiene que ver con la parte social del Estado ${ }^{8}$.

No obstante la anterior afirmación, la Corte Constitucional, en Sentencia C-228 de 2010, afirmó que el modelo económico que se adoptó en la Constitución Política fue el de economía social de mercado, con lo cual, a diferencia de lo anotado en el párrafo anterior, la libertad de empresa está limitada por la obligación de facilitar el cumplimiento de la protección del interés general, cuyo especial arraigo define la filosofía de un Estado Social. Afirmación que en consonancia con la Sentencia C-830 de 2010, debe entenderse que tal libertad, desde la óptica del control del Estado, no debe ser tal que sea nugatoria, sino que observe criterios de razonabilidad y proporcionalidad.

Tales aspectos son palpables con relativa facilidad a partir de la lectura del Título XII de la Carta, sobre el régimen económico de la hacienda pública, el cual, en su artículo 333, establece que la actividad económica y la iniciativa privada son libres; artículo cuyo valor debe entenderse tras la lectura de los artículos 58 y 60 superiores. Tal concepción supone el establecimiento de un rol más activo de parte de los ciudadanos, que como

TUCION\%20Y\%20LA\%20ECONOMIA\%20DE\% 20MERCADO\% 20-\%20SONIA\%20CORTES.pdf, visto el 2 de octubre de 2015 , p. 6.

6 Kalmanovitz, Salomón. Constitución y modelo económico. Conferencia dada en el seminario Diez años de la constitución colombiana. Facultad de Derecho Universidad Nacional, junio de 2001. Disponible en Internet: http://www.banrep.gov.co/docum/Lectura_finanzas/pdf/cmodelo.pdf.

7 Se toma como punto de partida el artículo 3 del GATT de 1947, el cual establece el Principio del Trato Nacional para las mercancías provenientes de otros países, principio que ha sido extendido hasta el área de los servicios y capitales, en virtud del Acuerdo General sobre el Comercio de Servicios; acuerdos estos de los que Colombia forma parte como miembro de la Organización Mundial del Comercio.

8 Uprimny, Rodrigo y ROdRíGueZ, CÉSAR. Constitución y modelo económico en Colombia: hacia una discusión productiva entre economía y derecho. Disponible en Internet: http://tic.uis.edu.co/ava/pluginfile.php/292578/ mod_resource/content/2/Constituci\%C3\%B3n_y_Modelo_Econ\%C3\%B3mico\%5B1\%5D\%20limpio. pdf, visto el 2 de octubre de 2015 . 
responsables de sus actos, son fundamentales para facilitar el crecimiento económico del Estado.

El citado artículo, a renglón seguido, anota que dichas libertades tendrán como único límite aquellos que la ley establezca; es decir que Colombia se convierte en un Estado interventor, a través de las diferentes disposiciones jurídicas que permitan el correcto desarrollo de un orden justo, dentro de la estructura del Estado Social de Derecho ${ }^{9}$. Sin embargo, tal aspecto es el que mayores dificultades ha supuesto, ya que el Estado no ha sido capaz de garantizar plenamente la satisfacción de los derechos fundamentales de las personas, y tampoco ha podido generar una política que sea realmente coherente con sus objetivos de crecimiento económico, y mucho menos garantizar a plenitud la igualdad entre las personas, ni entre los diferentes competidores en el mercado.

El Estado se enfrenta a la difícil tarea de permitir la libertad económica y, al mismo tiempo, mantener un rol intervencionista que no sea tal que, en últimas, termine por generar distorsiones a la economía, ni tensiones entre los diferentes competidores. Así, para garantizar la seguridad jurídica necesaria como gancho para la creación y establecimiento de nuevas empresas, debe generarse el clima perfecto, desde todas las ópticas, atendiendo a las necesidades no solo de los grandes capitales y de los consumidores, sino también de las medianas y pequeñas empresas que buscan posicionarse y lograr obtener así sea una pequeña porción del mercado.

El mantenimiento del derecho a la igualdad, como bandera de cualquier democracia participativa, requiere del funcionamiento de una economía de mercado que sirva como garantía de la institucionalidad propia del Estado y como interpretación de la teleología propia de la Carta Política ${ }^{10}$, para la correcta interacción de las distintas fuerzas que pujan para quedarse con los consumidores, $\mathrm{y}$, al mismo tiempo, para garantizar los derechos de estos últimos como la parte más débil dentro de la cadena.

\section{LA REGULACIÓN SOBRE PROTECCIÓN DE LA COMPETENCIA}

Con la promulgación de la Ley 155 de 1959, se abrió el paso a la protección de la competencia en Colombia. La misma, en su artículo primero, estableció una prohibición general sobre cualquier práctica restrictiva del comercio. Posteriormente, se expidió el Decreto 2153 de 1992, el cual, en primera instancia, tiene por objetivo principal la reestructuración de la Superintendencia de Industria y Comercio, de tal manera que la misma se ajustara a la Constitución Política de 1991 y pudiera asumir un rol más activo para alcanzar la meta de un mayor crecimiento económico, a través del establecimiento de un mejor desarrollo empresarial y garantizando, al mismo tiempo, el ejercicio de una sana competencia entre empresarios y protegiendo a los consumidores.

Igualmente, este Decreto evoluciona respecto de la Ley 155 de 1959, en la cual se realizó una prohibición general, y en su lugar se establecen descripciones de conductas 
específicas que pueden afectar la competencia, las cuales pueden ser objetiva o subjetivamente realizadas, y por último, pero no menos importante, manteniendo la postura ex ante frente a la comisión de las conductas, es decir que no se requiere demostrar la existencia de perjuicios para que se adelante un proceso o se adopten medidas cautelares.

Tales aspectos actualmente se encuentran regulados en la Ley 1340 de 2009, la cual modifica y adiciona las normas anteriormente citadas, y además concede competencia exclusiva a la Superintendencia de Industria y Comercio para que conozca de las investigaciones por violaciones a la libre competencia asociadas con procedimientos de integraciones empresariales.

Por lo tanto, aunque las normas que han regulado el tema, incluyendo el Decreto 2153 de 1992, no hagan ninguna referencia puntual a la existencia de monopolios, como sí ocurre con el artículo 336 Superior, tipifican cualquier abuso de la posición dominante como contrario al correcto comportamiento del mercado, no obstante que no debe entenderse como sinónimo de que no siempre el abuso de la posición dominante supone la existencia de un monopolio ${ }^{11}$.

Teniendo en cuenta lo anterior, y como mecanismo para garantizar la protección de la competencia, se prevé que en el caso de las integraciones empresariales que reúnan unas determinadas condiciones, como se verá más adelante, estas deberán ser notificadas a la SIC, para que de oficio analice la viabilidad o no de la autorización de estas.

\section{LA INTEGRACIÓN EMPRESARIAL}

La globalización, la búsqueda de la máxima de eficiencia y un mercado que es cada vez más exigente de productos y servicios han obligado a que las empresas cambien su tradicional visión del mercado y los paradigmas existentes, y en su lugar se orienten a la realización de uniones estratégicas con otras empresas, de manera que se facilite la obtención de resultados positivos, los cuales constituyen la principal razón de ser de cualquier sociedad comercial.

Dichas uniones, que pueden ser de carácter nacional o internacional, en forma de simples fusiones o adquisiciones, constituyen la base principal de lo que hoy se conoce como integraciones empresariales, con lo cual se logra un mayor grado de especialización y, a su vez, se garantiza la competitividad en un mercado que, como se anotó, es cada vez más exigente. La integración empresarial puede ser entendida entonces como una alianza que se realiza con el fin de ser más eficiente $y$, de esta forma, conseguir resultados en materia de competitividad y economía que no se lograrían si se actuara de forma aislada.

Tal posibilidad se previó por primera vez a través de la Ley 155 de 1959, la cual, en el parágrafo único de su artículo primero, estableció una excepción a la prohibición general, al permitir la realización de acuerdos o convenios siempre que se verificara que

11 Hernández, Diana LuCía. "Excepción de empresa en crisis. Ideas generales sobre la regulación de la figura en el derecho comparado y su aplicación en Colombia", en Revista E-Mercatoria. Enero-junio, 2014, vol. 13, n. $^{\circ} 1$. 
los mismos tuvieran como objetivo defender la estabilidad de un sector básico o el interés general de la economía.

La Ley 1340 de 2009, por medio de la cual se dictan normas en materia de protección de la competencia, y que a su vez modificó la Ley 155 de 1959 y el Decreto 2351 de 1992 , no define con precisión qué debe entenderse por integración empresarial. Empero, en su artículo noveno, establece un criterio de carácter subjetivo y otro de carácter objetivo, que en caso de presentarse en una operación de integración, las empresas estarán en la obligación de notificar a la Superintendencia de Industria y Comercio.

En primer lugar, el criterio subjetivo, el cual se configura bien sea cuando las empresas intervinientes se dedican a la misma actividad económica o cuando las empresas se encuentran en la misma cadena de valor, aspecto que es recogido igualmente en la Resolución 10930 del 11 de marzo de 2015 de la Superintendencia de Industria y Comercio. En segundo lugar, el criterio objetivo, a saber, haber obtenido individual o conjuntamente ingresos operacionales por valor $100.000 \mathrm{SMLMV}$, o tener activos totales superiores a $100.000 \mathrm{SMLMV}_{\text {; }}$ para ambos casos, la medición será realizará en el año inmediatamente anterior.

El mismo artículo establece que no obstante presentarse las condiciones antes señaladas, si la participación de las sociedades integradas en el mercado no es superior al $20 \%$, se entenderá autorizada la operación. Es posible entender que una participación mayor a dicho porcentaje podría ser entendida como la asunción de una posición de control en el mercado, entendido como reza el numeral 4 del artículo 45 del Decreto 2153 de 1992, como "la posibilidad de influenciar directa o indirectamente la política empresarial, la iniciación de los bienes o derechos esenciales para el desarrollo de la actividad de la empresa".

Por lo tanto, y de acuerdo con el Concepto 00001365 del 2 de marzo de 2000 de la SIC, no es de interés como tal la forma de integración, la figura que se utilice, sino el resultado de dicha actuación, en la medida en que pueda producir efectos sobre el normal y correcto funcionamiento del mercado. Se puede observar que se tiene de presente con mayor relevancia el criterio económico, y se flexibiliza el criterio jurídico ${ }^{12}$. Predominio que a la postre puede resultar contraproducente, en la medida en que se pueden afectar los principios constitucionales y la dificultad de poder garantizar el derecho a la igualdad para todos los operarios del mercado, lo cual, en últimas, supone una gran pérdida de seguridad jurídica ${ }^{13}$.

Hasta este punto, puede afirmarse que las integraciones empresariales no son ilegales; las mismas se encuentran cobijadas bajo el marco regulatorio colombiano, que responde a las previsiones del artículo 333 de la Carta Política, y como plena manifestación de la

12 SÁNCHEZ PACHÓN, LUIS. "La integración y colaboración intercooperativas en el marco del derecho de defensa de la competencia", en AA.VV., La aplicación privada del derecho de la competencia. Valladolid: Lex Nova, Unión Europea, Gobierno de España, Instituto de Estudios Europeos, 2011, p. 943.

13 Véase, en este mismo sentido, a Folguera Crespo, Jaime y Vidal Martínez, Patricia. Comentario a la Ley de defensa de la competencia. Madrid: Thomson-Civitas, 2006. 
autonomía de la voluntad privada; pero que al mismo tiempo, y en atención al mismo artículo de la Carta, deben someterse a la vigilancia y aprobación de la SIC en aquellos casos en que por encontrarse por encima de ciertos límites fijados por ley, puedan terminar por afectar la libre competencia ${ }^{14}$.

Así, de acuerdo con la Resolución n. ${ }^{\circ} 19110$ de julio 7 de 2003, puede decirse que el control que realiza la Superintendencia de Industria y Comercio a las integraciones -o concentraciones-, se configura como un elemento netamente preventivo, usado por esta para anticiparse a los posibles efectos negativos que pueda tener para la economía una operación de integración, y de esta forma evitar cualquier distorsión que esta pueda aparejar.

\section{LA EXCEPCIÓN DE EFICIENCIA}

Es de gran aceptación el hecho de que en determinadas circunstancias, las autoridades administrativas encargadas de los aspectos económicos de los Estados puedan modificar o establecer flexibilizaciones a las políticas de competencia, como mecanismo para facilitar la reactivación económica o garantizar el crecimiento nacional y la defensa de los consumidores. Acogiendo dicha postura, el artículo 12 de Ley 1340 de 2009 introdujo una modificación al artículo 51 del Decreto 2153 de 1992, el cual quedó de la siguiente manera:

Artículo 12. Excepción de Eficiencia. Modifíquese el artículo 51 del Decreto 2153 de 1992, el cual quedará así:

La autoridad nacional de competencia podrá no objetar una integración empresarial si los interesados demuestran dentro del proceso respectivo, con estudios fundamentados en metodologías de reconocido valor técnico que los efectos benéficos de la operación para los consumidores exceden el posible impacto negativo sobre la competencia $y$ que tales efectos no pueden alcanzarse por otros medios.

En este evento deberá acompañarse el compromiso de que los efectos benéficos serán trasladados a los consumidores. La Superintendencia de Industria y Comercio podrá abstenerse objetar una integración cuando independiente de la participación en el mercado nacional de la empresa integrada, las condiciones del mercado externo garanticen la libre competencia en el territorio nacional.

Parágrafo $1 .^{\circ}$. Cuando quiera que la autoridad de competencia se abstenga de objetar una operación de integración empresarial con sustento en la aplicación de la excepción de eficiencia, la autorización se considerará condicionada al comportamiento de los interesados, el cual debe ser consistente con los argumentos, estudios, pruebas y compromisos presentados para solicitar la aplicación de la excepción de eficiencia. La autoridad podrá exigir el otorgamiento de garantías que respalden la seriedad y el cumplimiento de los compromisos así adquiridos.

14 Véase, en este mismo sentido, a VAlEnCia Agudelo, Germán Dario. "Límites al crecimiento de la empresa en Colombia: un análisis neoinstitucional al sector de la generación eléctrica", en Revista AdMinister, Universidad Eafit. Enero-julio, 2006, n. ${ }^{\circ} 8$. 
Parágrafo 2..$^{\circ}$. En desarrollo de la función prevista en el número 21 del artículo $2^{\circ}$ del Decreto 2153 de 1992, la autoridad de competencia podrá expedir las instrucciones que especifiquen los elementos que tendrá en cuenta para el análisis y la valoración de los estudios presentados por los interesados.

En primera instancia, es posible afirmar que el artículo transcrito no ofrece mayor dificultad en cuanto a su interpretación. Se podrán realizar integraciones empresariales, sin oposición de ninguna autoridad, en aquellos casos en los que se pruebe que los beneficios que dicha integración arroje sobre los consumidores excederán el impacto negativo sobre la competencia.

Parafraseando a Schumpeter, se trata de la valoración de los procesos de destrucción creativa, en los que más allá de los empleos que se puedan destruir, debe valorarse el beneficio general que se generará ${ }^{15}$. Se trata, en consonancia con las previsiones de la Carta, de la anteposición de un interés general, de un grupo mayoritario de consumidores, sobre el interés particular de un empresario que se pueda ver afectado con dicha integración. Dicho en otras palabras, es una flexibilización normativa a situaciones que, por su naturaleza, merezcan un trato especial ${ }^{16}$.

En este caso, uno de los aspectos que no se puede discutir es que desde la configuración misma de la autorización, se sabe que esta será generadora de una restricción indebida a la competencia, ya que, como su nombre lo indica, se trata de una excepción. Ya que de acuerdo con Archila Peñalosa, si dentro del mercado los competidores se encuentran en similares condiciones de competencia, no existiera la necesidad de solicitar la excepción ${ }^{17}$.

Si bien no estamos en desacuerdo con una solución como la que plantea el citado artículo, creemos que la redacción del mismo es un tanto desafortunada, al no establecer, o al no haber sido reglamentados por ningún decreto, cuáles son los procedimientos y criterios objetivos que se valorarán para determinar si efectivamente se logra el cometido de mejorar las condiciones de acceso a un producto o servicio a un grupo de personas que sea lo suficientemente alto como para considerar que efectivamente se está generando un beneficio considerable.

Así, por ejemplo, el artículo requiere que se trate de un estudio de reconocido valor técnico, mas no hace referencia a cuáles son tales estudios, cuyo criterio puede variar de persona a persona; igualmente, cuando hace referencia a que debe demostrarse que el beneficio excede el impacto negativo, no se estableció un margen de medición.

En el parágrafo primero se establece la posibilidad de requerir garantías o no, comportamiento que también afectaría el derecho a la igualdad; teniendo en cuenta que se trata de una situación que puede afectar la competencia, consideramos que el requerimiento Aguilar S.A., 1952.

16 HeRnández, Diana LuCía.

17 Archila Peñalosa, Emilio José. "Novedades de la Ley 1340 de 2009 para el régimen de protección de la competencia", en Revista de Derecho y Economía, Universidad Externado, 2010, n. ${ }^{\circ} 32$. 
de tales garantías debería ser obligatorio para todas aquellas empresas que decidan integrarse y que soliciten que se les aplique la excepción de eficiencia.

Una de las dificultades que surge en este punto está dada porque si bien los estudios matemáticos y económicos siempre constituyen una herramienta de vital valor e importancia para la adopción de medidas de carácter económico para el desarrollo del país, no siempre sus resultados son tan atinados como se esperaría en primera instancia, teniendo en cuenta lo impredecible del mercado y del comportamiento humano.

Hasta aquí puede decirse, entonces, que esta tendencia creciente de los diferentes Estados a buscar la máxima eficiencia económica de sus mercados, para garantizar el crecimiento y desarrollo, debe observarse de forma concomitante con las normas que regulan la competencia, para no generar desigualdades en el funcionamiento de los mercados no solo a nivel nacional, sino también internacional ${ }^{18}$.

\section{CRÍTICA}

Se trata de un criterio de interés global que no debe tener en cuenta únicamente el aspecto económico, sino que debe ubicar al consumidor en medio de dicha relación, a efectos de poder verificar, lo más objetivamente posible, si para este sector la excepción podría ser realmente útil. De acuerdo con Khemani, debería afirmarse entonces que por cada unidad monetaria que reciban los empresarios a los que se les haya concedido una excepción, debe existir igualmente una unidad en manos de los consumidores ${ }^{19}$. Sin embargo, la realidad respecto a este tipo de prácticas, si bien no son comunes, puede terminar siendo otra.

Al identificarse este tipo de situaciones, el Estado, que es el principal garante del cumplimiento de la Constitución Política, y al mismo tiempo generador de las reglas de competencia para lograr la mayor eficiencia del mismo, debe generar políticas que garanticen a todos los competidores por igual su competitividad en el mercado, y no que generen desconfianza, reduciendo la iniciativa empresarial y las oportunidades de negocio $^{20}$.

Las asimetrías existentes entre las grandes y las pequeñas empresas son sentidas con mayor peso por las pequeñas empresas, lo cual aunque se encuentre establecido en la norma como una práctica legal, puede ser constitutivo de competencia desleal, máxime en escenarios en los que los beneficiados con dicha excepción puedan igualmente esta-

18 EhLERMANN, Claus-DieTER y LAUDATI, LARAINE. European competition law annual 1997, the objetives of competition policy. Oxford: Hard Publishing, 1998.

19 KhEmani, R. Shyam. "Aplicación de las leyes sobre la competencia: Exenciones y Excepciones", en Conferencia de las Naciones Unidas sobre comercio y desarrollo. Nueva York, 2002. Disponible en Internet: http:// unctad.org/es/Docs/ditcclpmisc25_sp.pdf, visto el 17 de octubre de 2015.

20 ZeVallos, Emilio. Restricciones del entorno a la competitividad empresarial en América Latina. Costa Rica: Editorial Fundes, 2007. 
blecer las reglas de juego a las que tendrán que acogerse los minoristas y distribuidores, así como las condiciones para nuevos participantes que quieran entrar al mercado ${ }^{21}$.

Lo anterior supone el caldo de cultivo para uno de situación que siempre ha sido una preocupación para el Estado, como es el empleo informal y la generación de empleo formal. Sin contar que si bien en primera instancia puede valorarse que exista un beneficio para el consumidor, el grupo empresarial beneficiado con la excepción tiene la facultad de establecer precios, que de ser muy altos, pueden disminuir la satisfacción y la causa que dio origen a la excepción, lo cual sería una situación de ineficiencia ${ }^{22}$.

La excepción de eficiencia debe tener en cuenta igualmente que la eficiencia no es el único criterio que debe evaluarse dentro de una economía, y mucho menos en una economía como la colombiana, que de acuerdo con su ordenamiento constitucional, se trata de un Estado Social de Derecho, en el cual se deben garantizar diferentes derechos, entre ellos la equidad y la igualdad.

Por lo tanto, para que a través del legislativo y del propio ordenamiento jurídico se pueda garantizar la eficiencia, se precisa, de una parte, el análisis exhaustivo que dicha figura tiene para determinar a ciencia cierta unos valores en términos de eficiencia, y al mismo tiempo, su articulación con los aspectos jurídicos, lo cual puede decirse que, en primera instancia, no se logra con la redacción del citado artículo 12.

Como ya se ha visto en el presente artículo, si bien el Estado colombiano, en virtud de su Constitución Política, debe jugar un rol bastante activo para lograr mantener el equilibrio y la libertad económica, consideramos oportuna la posición de Krugman y Wells al anotar que el Estado debe realizar su función de vigilancia más que buscar leyes para buscar la eficiencia, toda vez que es la misma mano invisible del mercado la que alcanza su punto de equilibrio. ${ }^{23}$.

Es decir, toda vez que no es tarea fácil que las fuerzas del mercado logren un punto de equilibrio, y que dicho equilibrio muchas veces es el resultado de distorsiones en la competencia entre las diferentes fuerzas que actúan en la economía, se precisa que el Estado actúe ${ }^{24}$, máxime en un sistema como el colombiano, que de conformidad con la Constitución Política de 1991, se estableció como Estado Social de Derecho, y por lo cual surge con mayor fuerza la obligación de poder garantizar la igualdad para todos los competidores.

\section{CONCLUSIONES}

Es evidente que no obstante que dentro del orden de la Carta Política, los derechos fundamentales se encuentran en primacía sobre los derechos económicos, para el Estado es

21 MARKOVITS, RICHARD. Economics and the interpretation and application of U.S. and E.U. Antitrust Law. Nueva York: Springer, 2014. vol. II.

22 Samuelson, Paul y Nordhaus, William. Economía. 18 ed. Madrid: Mc Graw Hill, 2005.

23 Krugman, Paul y Well, Robin. Macroeconomics. Nueva York: Worth Publishers, 2006.

24 Stiglitz, Joseph. La economía del sector público. 2. ${ }^{a}$ ed. Barcelona: Bosh Editores, 1995. 
de mayor importancia el desarrollo de los segundos, toda vez que de esta forma puede garantizar el crecimiento, desarrollo y el cumplimiento de los fines sociales previstos en la Constitución.

Empero, si bien una postura como la señalada no puede tacharse de negativa a primera instancia, se requiere que la misma se realice en observancia concomitante con todas las previsiones de la Carta, a efectos de no generar ni distorsiones en la economía, ni afectaciones a las garantías fundamentales de rango constitucional.

Una forma de mejorar el crecimiento sin generar mayor riesgo sería un mayor estudio de las diferentes normas jurídicas que se desarrollan, a efectos de que las mismas sean realmente una respuesta a los problemas que enfrenta el país, garantizando la igualdad, la justicia y la seguridad jurídica para poder generar certeza entre los diferentes operadores del mercado.

Lo anterior supone que las normas que se promulguen en el país observen no solo los aspectos económicos, sino también las diferentes consecuencias jurídicas. En tal sentido, un avance sería el establecimiento de procedimientos unificados, que no se dejen a criterio del funcionario de turno, ya que ello facilita los descontentos, así como las diferentes interpretaciones, y más perjudicial aún, la corrupción.

En la medida en que el Estado se concentre en la realización correcta de sus funciones, como garante e interventor de la economía, las integraciones económicas facilitarán realmente el crecimiento económico y no supondrán una amenaza a la libre competencia, como ha ocurrido con los casos recientes que han sido sancionados por la SIC, en los cuales se observa un evidente abuso de la posición dominante.

BIBLIOGRAFÍA

Aragón Reyes, Manuel. Libertades económicas y estado social. Madrid: McGraw Hill, 1995.

ArChila Peñalosa, Emilio José. "Novedades de la Ley 1340 de 2009 para el régimen de protección de la competencia", en Revista de Derecho y Economía, Universidad Externado. 2010, n. ${ }^{\circ} 32$.

Cortés Zambrano, Sonia Patricia. "El estado, la constitución y la economía de mercado", en Revista virtual vía inviniendi et iudicandi, Universidad Santo Tomás. Disponible en Internet: http:// numanterioresviei.usta.edu.co/articulos/edi9/04-Sonia-Patricia-Cortez-Zambrano/EL\%20 ESTADO,\%20LA\%20CONSTITUCION\%20Y\%20LA\%20ECONOMIA\%20DE\%20MERCADO\% 20-\%20 SONIA\%20CORTES.pdf, visto el 2 de octubre de 2015, p. 6.

Ehlermann, Claus-Dieter y LaUdaTI, LaRAine. European competition law annual 1997, the objetives of competition policy. Oxford: Hard Publishing, 1998.

Folguera Crespo, Jaime y Vidal Martínez, Patricia. Comentario a la Ley de defensa de la competencia. Madrid: Thomson-Civitas, 2006.

HernándeZ, Diana LuCía. "Excepción de Empresa en crisis. Ideas generales sobre la regulación de la figura en el derecho comparado y su aplicación en Colombia", en Revista E-Mercatoria. Enero-junio, 2014. vol. 13, n. ${ }^{\circ}$. 
Kalmanovitz, Salomón. Constitución y modelo económico. Conferencia dada en el seminario Diez años de la constitución colombiana, Facultad de Derecho Universidad Nacional, junio de 2001. Disponible en Internet: http://www.banrep.gov.co/docum/Lectura_finanzas/pdf/cmodelo.pdf.

Khemani, R. Shyam. "Aplicación de las leyes sobre la competencia: exenciones y excepciones", en Conferencia de las Naciones Unidas sobre comercio y desarrollo. Nueva York, 2002. Disponible en Internet: http://unctad.org/es/Docs/ditcclpmisc25_sp.pdf, visto el 17 de octubre de 2015

Krugman, Paul y Well, Robin. Macroeconomics. Nueva York: Worth Publishers, 2006.

MARKOVITS, RICHARD. Economics and the interpretation and application of U.S. and E. U. Antitrust Law. Nueva York: Springer, 2014. vol. II.

Miranda Londoño, Alfonso. "Origen y evolución del derecho de la competencia en Colombia, la Ley 155 de 1959 y su legado", en Revista del Derecho de la Competencia. Enero-diciembre, 2011, vol. 6, n. $^{\circ} 6$, pp. $65-148$

Samuelson, Paul y Nordhaus, William. Economía. 18 ed. Madrid: Mc Graw Hill, 2005.

SÁNCHEZ PACHÓN, LUIS. "La integración y colaboración intercooperativas en el marco del derecho de denfesa de la competencia", en AA.VV., La aplicación privada del derecho de la competencia. Valladolid: Lex Nova, Unión Europea, Gobierno de España, Instituto de Estudios Europeos, 2011, p. 943.

SCHUMPETER, Joseph. Capitalismo, socialismo y democracia (José DíAz García trad.). México: Ediciones Aguilar S.A., 1952.

STIGLiTZ, JosePH. La economía del sector público. 2. ${ }^{\text {a }}$ ed. Barcelona: Bosh Editores, 1995.

Uprimny, Rodrigo y RodríGUeZ, CésAR. Constitución y modelo económico en Colombia: bacia una discusión productiva entre economía y derecho. Disponible en internet: http://tic.uis.edu.co/ ava/pluginfile.php/292578/mod_resource/content/2/Constituci\%C3\%B3n_y_Modelo_ Econ\%C3\%B3mico\%5B1\%5D\%20limpio.pdf, visto el 2 de octubre de 2015.

Valencia Agudelo, Germán Dario. "Límites al crecimiento de la empresa en Colombia: un análisis neoinstitucional al sector de la generación eléctrica", en Revista Ad-Minister, Universidad Eafit. Enero-julio, 2006, n. ${ }^{\circ} 8$.

Zevallos, EMILIO. Restricciones del entorno a la competitividad empresarial en América Latina. Costa Rica: Editorial Fundes, 2007.

\section{Legislación y jurisprudencia}

Constitución Política de Colombia

Decreto 2153 de 1992

Ley 155 de 1959

Ley 1340 de 2009

Superintendencia de Industria y Comercio, Resolución n. ${ }^{\circ} 19110$ del 7 de julio de 2003.

Superintendencia de Industria y Comercio, Resolución n. ${ }^{\circ} 10930$ del 11 de marzo de 2015.

Corte Constitucional Colombiana, Sentencia C-074 de 1993.

Corte Constitucional Colombiana, Sentencia C-228 de 2010. 